\title{
Effect of microslit retention on the bond strength of zirconia to dental materials
}

\author{
Shogo IWAGURO'1, Saiji SHIMOE², Isao HIRATA², Takeshi MURAYAMA ${ }^{4}$ and Takahiro SATODA ${ }^{5}$ \\ ${ }^{1}$ Division of Dental Technician, Department of Clinical Practice and Support, Hiroshima University Hospital, 2-3, Kasumi 1-Chome, Minami-ku, \\ Hiroshima 734-8551, Japan \\ ${ }^{2}$ Department of Anatomy and Functional Restorations, Oral Health Sciences, Hiroshima University Graduate School of Biomedical Sciences, 2-3, \\ Kasumi 1-Chome, Minami-ku, Hiroshima 734-8553, Japan \\ ${ }^{3}$ Department of Biomaterials, Basic Life Sciences, Hiroshima University Graduate School of Biomedical Sciences, 2-3, Kasumi 1-Chome, Minami-ku, \\ Hiroshima 734-8553, Japan \\ ${ }^{4}$ Department of Medical System Engineering, Oral Health Sciences, Hiroshima University Graduate School of Biomedical Sciences, 2-3, Kasumi \\ 1-Chome, Minami-ku, Hiroshima 734-8553, Japan \\ ${ }^{5}$ Department of Rehabilitation, Hiroshima International University, 555-36, Kurosegakuendai, Higashihiroshima City, Hiroshima 739-2695, Japan \\ Corresponding author, Saiji SHIMOE; E-mail: shimoe@hiroshima-u.ac.jp
}

\begin{abstract}
The aim of this study was to investigate the effect of microslits formed by $\mathrm{Nd}: \mathrm{YVO}_{4}$ laser beam machining on the bond strength between two types of zirconia, yttria-partially stabilized zirconia (Y-TZP) and ceria-partially stabilized zirconia/alumina nanocomposite (CeTZP/A), and porcelain or two types of resin. Zirconia disks were divided into three groups: 1) non-treated (NT); 2) blasted with alumina particles $(\mathrm{AB})$; 3) microslits fabricated on a zirconia surface by laser beam machining (MS). After veneering porcelain or resins on zirconia specimens, halves of the resin specimens were thermocycled up to 20,000 cycles. The shear bond strength between porcelain and both types of zirconia was not improved by the microslits. Before and after thermocycling, the bond strength between an indirect composite resin or acrylic resin and Y-TZP with microslits was the highest. It was concluded that the microslits on Y-TZP enabled micromechanical interlocking and improved the bond strength and durability of the resins.
\end{abstract}

Keywords: Bond strength, Zirconia, Microslit, Porcelain, Resin

\section{INTRODUCTION}

In the traditional methods of restorative dentistry, metal was widely used in combination with resin or ceramic. Contrary to this, zirconia has been used in recent years as monolithic anatomically contoured crown or corematerials for ceramic crown in restorative dentistry. Zirconia has advantages such as high fracture toughness, flexural toughness and better aesthetic appearance, which makes it a viable alternative to metal.

A representative example of zirconia use in combination with other materials is zirconia-supported ceramic restoration. The use of a zirconia frame for fixed partial dentures has excellent aesthetics that are better than a metal frame because zirconia is a white colored material in the normal state. However, there are some problems of fracturing and dislodgement, although such an all-ceramic must provide longevity similar to metalceramic composites ${ }^{1,2)}$.

It is also possible that indirect composite resins be applied to a zirconia frame in the fabrication of resin facing restorations. Indirect composite resins are easily manipulated chair-side and in the laboratory, and can be repaired in the oral cavity. The wear resistance of indirect composite resins has also been improved by the use of fillers ${ }^{3}$. Considering that porcelain is a brittle material, the demand for zirconia-composite restorations may increase in the future.

Moreover, with the advance in dental computeraided design and computer-aided manufacturing (CAD/ CAM) systems, the fabrication of removable dentures that contain zirconia frameworks, bars, and clasps has become possible ${ }^{4,5)}$. Although removable partial dentures with zirconia frameworks have room for improvement for clinical use, it is anticipated that a combination of zirconia dentures and acrylic resin will be used in the future because of the biocompatibility and corrosion resistance of these materials. Therefore, it is important to establish the strength and stability of the adhesive bond between zirconia and acrylic resin.

In the bonding between zirconia and each material, improvement of the bond strength plays an important role in the component longevity. For example, some primers are effective to improve bond strength between resin and zirconia $^{6-9)}$. Nevertheless, when considering a specific of resin, mechanical interlocking is required for bond stability. It is expected that mechanical interlocking is one of the important methods to achieve strong adhesion. In resin-metal restoration, the use of retention beads is a popular method used to achieve this purpose by providing undercuts ${ }^{10,11)}$. However, milling systems are not able to manufacture such microstructure. Although there have been a number of reports concerning zirconia surfaces roughened by alumina blasting ${ }^{7,12-14)}$, acid etching ${ }^{15)}$, or porous structure ${ }^{16)}$, there is little information available on bonding zirconia with a regular arrangement microstructure to other composite materials.

Another approach is laser machining, where it is unnecessary to consider consumption of burs, and fine processing can be accurately performed. There have been a number of studies on laser irradiation of zirconia. Henriques et al. ${ }^{17)}$ investigated the strength 
of the bond between zirconia and porcelain following structuring of the zirconia surface using neodymiumdoped yttrium aluminum garnet (Nd:YAG) laser irradiation. They found that laser structuring increased the shear bond strength (SBS) by up to $75 \%$. In addition, they reported that no phase transformation occurred as a result of laser heating. On the other hand, Noda et $a l .{ }^{18)}$ stated that $\mathrm{Nd}$ :YAG dental laser irradiation is not suitable for welding zirconia because it induces surface cracking. Machining using a neodymium-doped yttrium orthovanadate $\left(\mathrm{Nd}: \mathrm{YVO}_{4}\right)$ laser has also been reported ${ }^{19}$. It was concluded that an industrial $\mathrm{Nd}$ : $\mathrm{YVO}_{4}$ laser was capable of fabricating a coping directly from fully sintered yttria-partially stabilized zirconia (Y-TZP). Such lasers have a short pulse width and a power density that is several hundred times higher than that for a dental $\mathrm{Nd}$ :YAG laser. It is therefore considered possible that the strength of the bond between zirconia and veneering materials could be improved by fabricating microslits in the zirconia using a computerized numerical control (CNC) $\mathrm{Nd}: \mathrm{YVO}_{4}$ laser machine. The purpose of the present study was to evaluate the effect of these microslits on the bond strength between two types of zirconia and porcelain or two types of resin.

\section{MATERIALS AND METHODS}

Specimen preparation and surface treatment

The materials used in this study are presented in Table 1. Two types of tetragonal zirconia polycrystal (TZP), $\mathrm{Y}-\mathrm{TZP}$ and ceria-partially stabilized zirconia/alumina nanocomposite (Ce-TZP/A), were used. Y-TZP (Cercon
Base, Degudent, Hanau-Wolfgang, Germany) and CeTZP/A (P-NANO ZR, Panasonic Healthcare, Tokyo, Japan) were sintered for $2 \mathrm{~h}$ at 1,350 and $1,450^{\circ} \mathrm{C}$, respectively, to produce disks with a diameter of $10 \mathrm{~mm}$ and a thickness of $2.5 \mathrm{~mm}$. One surface of the disks was polished with 600-grit silicon carbide abrasive paper.

Zirconia disks were divided into three groups according to the surface treatment performed: (1) nontreated (NT), (2) blasted with alumina particles (AB), and (3) with fabricated the microslits (MS). Aluminablasting of the $\mathrm{AB}$ group was performed on the zirconia surface with $50 \mu \mathrm{m}$ alumina particles (Cobra, Renfert, Hilzingen, Germany) for $10 \mathrm{~s}$ at a pressure of $0.3 \mathrm{MPa}$ and at a distance of $10 \mathrm{~mm}$ between the nozzle (Basic quattro, Renfert) and the surface. The microslits on the MS group samples were fabricated as reticular slits (width and pitch: $40 \mu \mathrm{m}$, depth: $25 \mu \mathrm{m}$ ) using a CNC laser machine (LASERTEC 40, DMG MORI, Nagoya, Japan). The laser medium was $\mathrm{Nd}: \mathrm{YVO}_{4}$, and the laser irradiation parameters were as follows: frequency of 70 $\mathrm{kHz}$, wavelength of $1,065 \mathrm{~nm}, 60 \mathrm{~mm}$ from the surface, and normal incidence. After laser machining, a thin surface layer became black because laser irradiation reduced oxygen content on the surface of zirconia ${ }^{18)}$. Since black color is aesthetically undesirable for clinical use, the specimens were then heated at a rate of $55^{\circ} \mathrm{C} / \mathrm{min}$ and held at $1,000^{\circ} \mathrm{C}$ for $5 \mathrm{~min}$ to recover oxygen ${ }^{17)}$ and restore white color. Figures 1 and 2 show SEM images of surface of Y-TZP and Ce-TZP/A before veneering.

Material veneering procedure

The procedure for firing porcelain to zirconia was

Table 1 Materials used for this study

\begin{tabular}{|c|c|c|c|}
\hline Material & Composition & Manufacturer & Lot No. \\
\hline \multicolumn{4}{|l|}{ Zirconia } \\
\hline Cercon Base (Y-TZP) & $\mathrm{ZrO}_{2}, \mathrm{Y}_{2} \mathrm{O}_{3}, \mathrm{HfO}_{2}, \mathrm{Al}_{2} \mathrm{O}_{3}, \mathrm{SiO}_{2}$ & Degudent, Hanau-Wolfgang, Germany & 18007813 \\
\hline P-NANO ZR (Ce-TZP/A) & $\mathrm{ZrO}_{2}, \mathrm{Al}_{2} \mathrm{O}_{3}, \mathrm{CeO}_{2}, \mathrm{HfO}_{2}$ & Panasonic Healthcare, Tokyo, Japan & G2222 \\
\hline \multicolumn{4}{|l|}{ Porcelain } \\
\hline Cercon Ceram Kiss & & Degudent & \\
\hline Paste Liner & Selenium, Feldspathic porcelain & & 91685 \\
\hline Dentin & Feldspathic porcelain & & 93629 \\
\hline \multicolumn{4}{|l|}{ Indirect composite resin } \\
\hline Gradia & & GC, Tokyo, Japan & \\
\hline Foundation Opaque & UDMA, $\mathrm{SiO}_{2}$ & & $130902 \mathrm{~B}$ \\
\hline Opaque & UDMA, $\mathrm{SiO}_{2}$ & & 1310152 \\
\hline Dentin & Filler, UDMA, $\mathrm{SiO}_{2}$, grass powder & & 1307171 \\
\hline \multicolumn{4}{|l|}{ Acrylic resin } \\
\hline PalaXpress ultra & & Kulzer, Hanau, Germany & \\
\hline Powder & PMMA, etc. & & 12030 \\
\hline Liquid & MMA, etc. & & 10223 \\
\hline \multicolumn{4}{|l|}{ Primer } \\
\hline Alloy primer & VTD, MDP, Acetone & Kuraray Noritake Dental, Tokyo, Japan & 190025 \\
\hline
\end{tabular}

UDMA=urethane dimethacrylate; PMMA=Polymethyl methacrylate; MMA=methyl methacrylate; VTD=6-(4-vinylbenzyl$n$-propyl)amino-1,3,5-triazine-2,4-dithiol, or -2,4-dithione tautomer; MDP=10-methacryloyloxydecyl dihydrogen phosphate; $\mathrm{PETP}=$ pentaerythritol tetrakis(3-mercaptopropionate) 

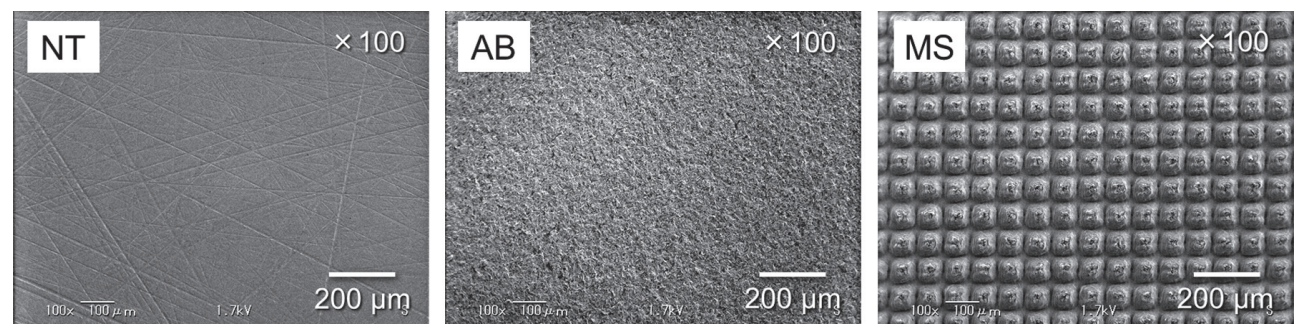

Fig. 1 SEM images of surface of Y-TZP before veneering (100×).
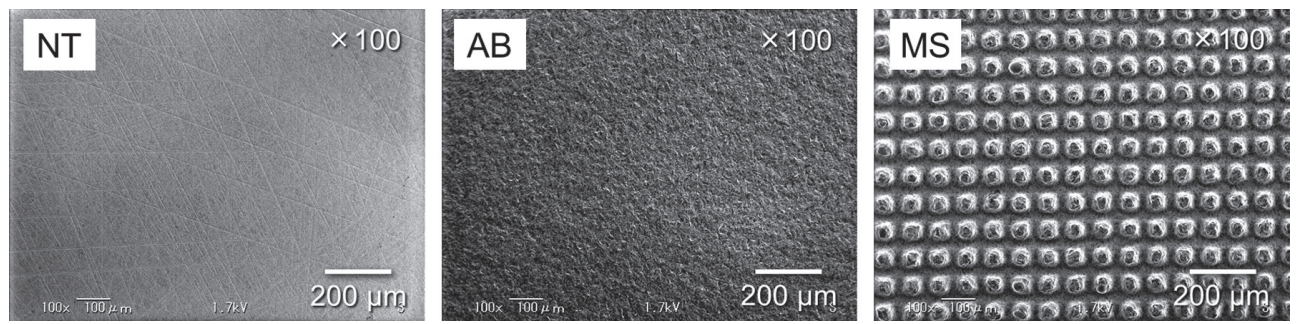

Fig. 2 SEM images of surface of Ce-TZP/A before veneering (100×).

Table 2 Firing schedule of porcelain

\begin{tabular}{|c|c|c|c|c|c|c|}
\hline & $\begin{array}{c}\text { Pre-heating } \\
\qquad\left({ }^{\circ} \mathrm{C}\right)\end{array}$ & $\begin{array}{l}\text { Drying time } \\
\text { pre-heating } \\
\quad(\min )\end{array}$ & $\begin{array}{l}\text { Heating rate } \\
\left({ }^{\circ} \mathrm{C} / \mathrm{min}\right)\end{array}$ & $\begin{array}{l}\text { End temp. } \\
\qquad\left({ }^{\circ} \mathrm{C}\right)\end{array}$ & $\begin{array}{l}\text { Holding time } \\
\qquad(\min )\end{array}$ & $\begin{array}{c}\text { Vacuum } \\
\text { (hPa) }\end{array}$ \\
\hline Paste liner 1 & 575 & 8:00 & 55 & 970 & $1: 00$ & 50 \\
\hline Paste liner 2 & 575 & 8:00 & 55 & 960 & $1: 00$ & 50 \\
\hline Dentin & 450 & $5: 00$ & 55 & 830 & $1: 30$ & 50 \\
\hline
\end{tabular}

conducted as follows. After a layer of liner material (Cercon Ceram Kiss, Degudent) was applied to the area defined by tape with a circular $5 \mathrm{~mm}$ diameter hole, the tape was removed from the surface of the specimen. The specimen was then transferred to a firing tray and fused in a porcelain furnace, according to the manufacturer's instructions given in Table 2. After firing the liner, dentin porcelain (Cercon Ceram Kiss, Degudent) was applied onto the liner surface using a cylindrical vinyl mold (6 mm internal diameter, $2.5 \mathrm{~mm}$ long, and $1 \mathrm{~mm}$ wall thickness). The mold was removed after drying. Dentin porcelains were fired in a porcelain furnace (Cerafusion VPF, J Morita, Osaka, Japan) according to the manufacturer's instructions given in Table 2 .

The procedure for bonding indirect composite resin to zirconia was conducted as follows. Primer (Alloy Primer, Kuraray Noritake Dental, Tokyo, Japan) was applied to the area defined by double-sided tape with a circular $5 \mathrm{~mm}$ diameter hole. Foundation opaque and opaque (Gradia, GC, Tokyo, Japan) resins were placed on the primed surface and exposed to light for $60 \mathrm{~s}$ in a lightcuring unit (Hi-light power, Kulzer, Hanau, Germany). Dentin shade indirect composite resin (Gradia, GC) was filled in a brass mold (6 $\mathrm{mm}$ internal diameter, $2 \mathrm{~mm}$ long, and $1 \mathrm{~mm}$ wall thickness) and photopolymerized for $180 \mathrm{~s}$ in the light-curing unit.
The procedure for bonding acrylic resin to zirconia was conducted as follows. After application of the primer to the zirconia surface in the same way as that for the indirect composite resin, denture base resin (PalaXpress ultra, Kulzer) was mixed at a powder/liquid ratio of 10 $\mathrm{g} / 7 \mathrm{~mL}$ and poured into a brass mold $(6 \mathrm{~mm}$ internal diameter, $2 \mathrm{~mm}$ long, and $1 \mathrm{~mm}$ wall thickness), then fixed by double-sided tape with a circular $5 \mathrm{~mm}$ diameter hole. The specimens were then polymerized in a pressure vessel (Palamat practic ELT, Kulzer) according to the manufacturer's instructions (Polymerization time: 30 min, water temperature: $55^{\circ} \mathrm{C}$, pressure: 2 bar).

All specimens were immersed in distilled water at $37^{\circ} \mathrm{C}$ for $24 \mathrm{~h}$. This state was defined as 0 thermocycles. Indirect composite resin and acrylic resin specimens were placed in a thermocycling apparatus (Thermal cycler, Nissin Seiki, Hiroshima, Japan) and cycled between 4 and $60^{\circ} \mathrm{C}$ in water with a 1 min dwell time per water bath for 20,000 cycles.

\section{Shear test and statistical analysis}

Specimens veneered with porcelain, indirect composite resin, and acrylic resin $(n=10)$ were seated in a shear testing jig during testing. Loads for shear tests were applied using a universal testing machine (AGS-5kNJ, Shimadzu, Kyoto, Japan) at a crosshead speed of 0.5 
$\mathrm{mm} / \mathrm{min}$. SBS was calculated by dividing the peak load $(\mathrm{N})$ by the bonded area. The values for each group were compared using the two-way analysis of variance (ANOVA) at a significance level of 0.05. After ANOVA, multiple comparisons were performed using the Scheffe's test. The statistical significance level was set at 0.05 . In addition, to analyze the influence of the thermocycling, the results for 0 and 20,000 cycles of an identical surface treatment were compared using Mann-Whitney's $U$ test with the value of statistical significance set at 0.05 for each treatment. All analyses were performed using SPSS 15.0 for Windows (SPSS Japan, Tokyo, Japan).

\section{Failure analysis}

After the shear bond tests, failure modes were categorized as adhesive failure at the zirconia-veneer material interface (A), cohesive failure at the inside of veneer material (C), or a mixture of adhesive and cohesive failure (AC). The bonding surfaces of specimens used for the shear bond test were observed using scanning electron microscopy (SE-8000, Keyence, Osaka, Japan) at a magnification of $500 \times$.

\section{RESULTS}

Table 3 shows the SBSs of porcelain veneered to zirconia. Among the Y-TZP specimens, the SBS ranged from a maximum of $26.2 \mathrm{MPa}$ in the MS group to a minimum of $23.4 \mathrm{MPa}$ in the NT group. However, there was no significant difference between groups. On the other hand, the SBS for Ce-TZP/A ranged from a maximum of $24.5 \mathrm{MPa}$ in the MS group to a minimum of $20.1 \mathrm{MPa}$ in the $\mathrm{AB}$ group. However, no statistical significant differences were found. Failure modes after shear tests are summarized in Table 4. The MS groups of both types of zirconia showed cohesive failure (C), and SEM observations of the specimens used for the shear test revealed that residual porcelain penetrates into the microslits of both MS groups (Figs. 3 and 4).

Table 5 shows the SBSs of indirect composite resin veneered to zirconia. For the mean SBS values of Y-TZP before and after thermocycling, the MS group was highest, and the $\mathrm{AB}$ group was significantly higher than the NT group. On the other hand, for the mean SBS values of $\mathrm{Ce}-\mathrm{TZP} / \mathrm{A}$, there was a significant difference among all groups and the $\mathrm{AB}$ group had the highest mean SBS followed by the MS and NT groups. Failure modes after shear tests are summarized in Table 6. No cohesive failure (C) was found in the NT and AB groups among both types of zirconia. For Y-TZP, the MS groups exhibited cohesive failure (C) both before and after the thermocycles. However, the MS group of Ce-TZP/A tended to exhibit mixed failure (AC) before the thermocycles and adhesive failure (A) after the thermocycles. SEM observations revealed that the microslits on Y-TZP were filled with resin before and after thermocycles; however, the resin in the other groups was either completely or partially debonded (Figs. 5 and 6).

Table 7 shows the SBSs of acrylic resin veneered to

Table 3 Shear bond strengths of porcelain veneered to zirconia

\begin{tabular}{cccc}
\hline Groups & Mean & SD & Category \\
\hline Y-TZP & & & \\
NT & 23.4 & 5.9 & $\mathrm{a}$ \\
AB & 24.9 & 7.0 & $\mathrm{a}$ \\
MS & 26.2 & 9.7 & $\mathrm{a}$ \\
Ce-TZP/A & & & \\
NT & 23.2 & 7.9 & $\mathrm{~A}$ \\
AB & 20.1 & 6.4 & $\mathrm{~A}$ \\
MS & 24.5 & 6.3 & $\mathrm{~A}$ \\
\hline
\end{tabular}

SD: Standard deviation; Category: Identical letters indicate that values are not statistically different $(p>0.05)$.

Table 4 Failure modes of veneered porcelain after shear bond testing

\begin{tabular}{cccc}
\hline Groups & A & AC & C \\
\hline Y-TZP & & 3 & 1 \\
NT & 6 & 8 & 0 \\
AB & 2 & 0 & 0 \\
MS & 0 & & 0 \\
Ce-TZP/A & & 8 & 0 \\
NT & 2 & 8 & 0 \\
AB & 2 & 0 & 10 \\
MS & 0 & 0 & 0 \\
\hline
\end{tabular}

A: Adhesive failure at the zirconia-porcelain interface; C: Cohesive failure within the porcelain; AC: Combination of adhesive and cohesive failures. 

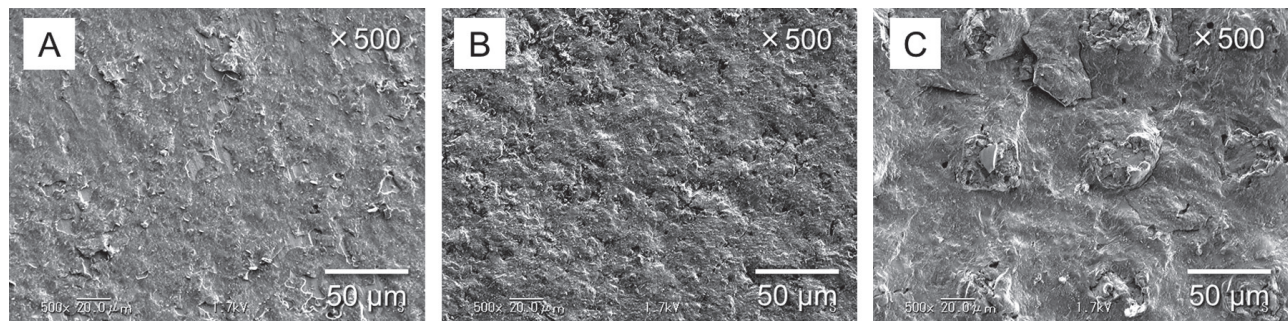

Fig. 3 SEM images of debonded surfaces of Y-TZP veneered with porcelain $(500 \times)$; (A) non-treated, (B) alumina-blasted, and (C) with fabricated microslits.
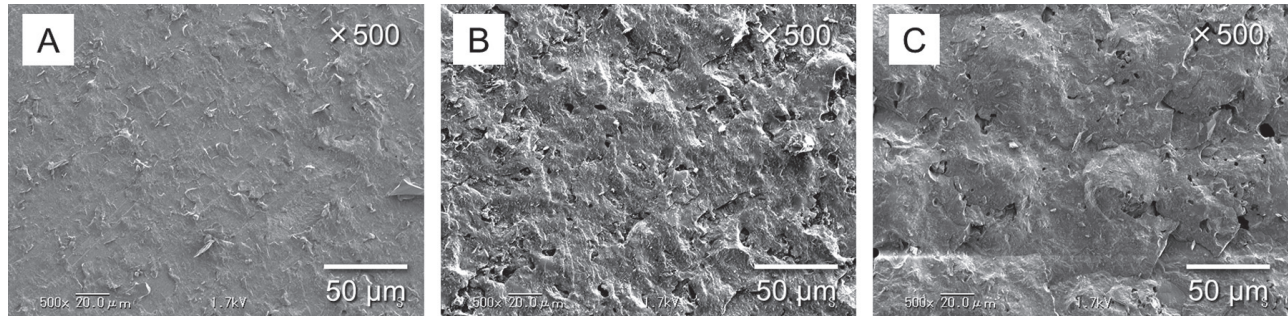

Fig. 4 SEM images of debonded surfaces of Ce-TZP/A veneered with porcelain $(500 \times)$; (A) non-treated, (B) alumina-blasted, and (C) with fabricated microslits.

Table 5 Shear bond strengths of indirect composite resin veneered to zirconia

\begin{tabular}{|c|c|c|c|c|c|c|c|c|}
\hline \multirow{2}{*}{ Groups } & \multicolumn{3}{|c|}{0 Thermocycles } & \multicolumn{3}{|c|}{ 20,000 Thermocycles } & \multirow{2}{*}{$\mathrm{S}$} & \multirow{2}{*}{ Reduction } \\
\hline & Mean & $\mathrm{SD}$ & Category & Mean & $\mathrm{SD}$ & Category & & \\
\hline \multicolumn{9}{|l|}{ Y-TZP } \\
\hline NT & 21.7 & 2.4 & a & 0 & 0 & & $\mathrm{~S}$ & $100 \%$ \\
\hline $\mathrm{AB}$ & 27.2 & 4.8 & $\mathrm{~b}$ & 27.9 & 4.4 & $d$ & & $-2.6 \%$ \\
\hline MS & 32.5 & 5.2 & $\mathrm{c}$ & 35.9 & 5.9 & $\mathrm{e}$ & & $-10.5 \%$ \\
\hline \multicolumn{9}{|l|}{$\mathrm{Ce}-\mathrm{TZP} / \mathrm{A}$} \\
\hline NT & 15.3 & 8.0 & $\mathrm{~A}$ & 0 & 0 & & $\mathrm{~S}$ & $100 \%$ \\
\hline $\mathrm{AB}$ & 30.0 & 3.7 & $\mathrm{C}$ & 24.6 & 3.7 & $\mathrm{D}$ & & $18.0 \%$ \\
\hline MS & 21.9 & 4.3 & $\mathrm{~B}$ & 13.6 & 6.5 & $\mathrm{E}$ & & $37.9 \%$ \\
\hline
\end{tabular}

SD: Standard deviation; Category: Identical letters indicate that values are not statistically different ( $p>0.05)$; S: Significant difference between pre- and post-thermocycling bond strengths $(p<0.05)$.

Table 6 Failure modes of indirect composite resin after shear bond testing

\begin{tabular}{|c|c|c|c|c|c|c|}
\hline \multirow{2}{*}{$\begin{array}{c}\text { Groups } \\
\text { Failure mode }\end{array}$} & \multicolumn{3}{|c|}{$\begin{array}{c}0 \\
\text { Thermocycles }\end{array}$} & \multicolumn{3}{|c|}{$\begin{array}{c}20,000 \\
\text { Thermocycles }\end{array}$} \\
\hline & A & $\mathrm{AC}$ & $\mathrm{C}$ & $\mathrm{A}$ & $\mathrm{AC}$ & $\mathrm{C}$ \\
\hline \multicolumn{7}{|l|}{ Y-TZP } \\
\hline $\mathrm{NT}$ & 0 & 10 & 0 & 10 & 0 & 0 \\
\hline $\mathrm{AB}$ & 3 & 7 & 0 & 0 & 10 & 0 \\
\hline MS & 0 & 0 & 10 & 0 & 0 & 10 \\
\hline \multicolumn{7}{|l|}{$\mathrm{Ce}-\mathrm{TZP} / \mathrm{A}$} \\
\hline NT & 2 & 8 & 0 & 10 & 0 & 0 \\
\hline $\mathrm{AB}$ & 1 & 9 & 0 & 0 & 10 & 0 \\
\hline MS & 0 & 8 & 2 & 8 & 2 & 0 \\
\hline
\end{tabular}

A: Adhesive failure at the zirconia-resin interface; C: Cohesive failure within the resin; AC: Combination of adhesive and cohesive failures. 
zirconia. As a result of shear testing before thermocycling, the MS group of Y-TZP exhibited significantly higher SBSs than the other groups; however, the MS group of Ce-TZP/A showed lower SBSs than the NT group. After thermocycling, the SBSs of both zirconia specimens, except the MS group of Y-TZP, could not be evaluated, because the resin was already debonded before the tests. Table 8 presents the distribution of the failure modes in the groups. The MS group of Y-TZP tended to exhibit mixed failure (AC) before and after thermocycling, although the other groups of both zirconia types exhibited adhesive failure. SEM observations after the tests revealed that the acrylic resin remained partially in microslits of Y-TZP (Figs. 7 and 8).

\section{DISCUSSION}

Mechanical interlocking is one of the factors that determines the bond strength between a core material and other materials. For example, improvement of the adhesive strength through the use of retention beads is widely employed for metal-composite restoration ${ }^{10,11,20)}$. However, many zirconia frames are manufactured using a milling system, and the minimum diameter of milling burs for dental CAD/CAM systems is $0.4-0.6 \mathrm{~mm}$. Therefore, it is impossible to produce slits with widths
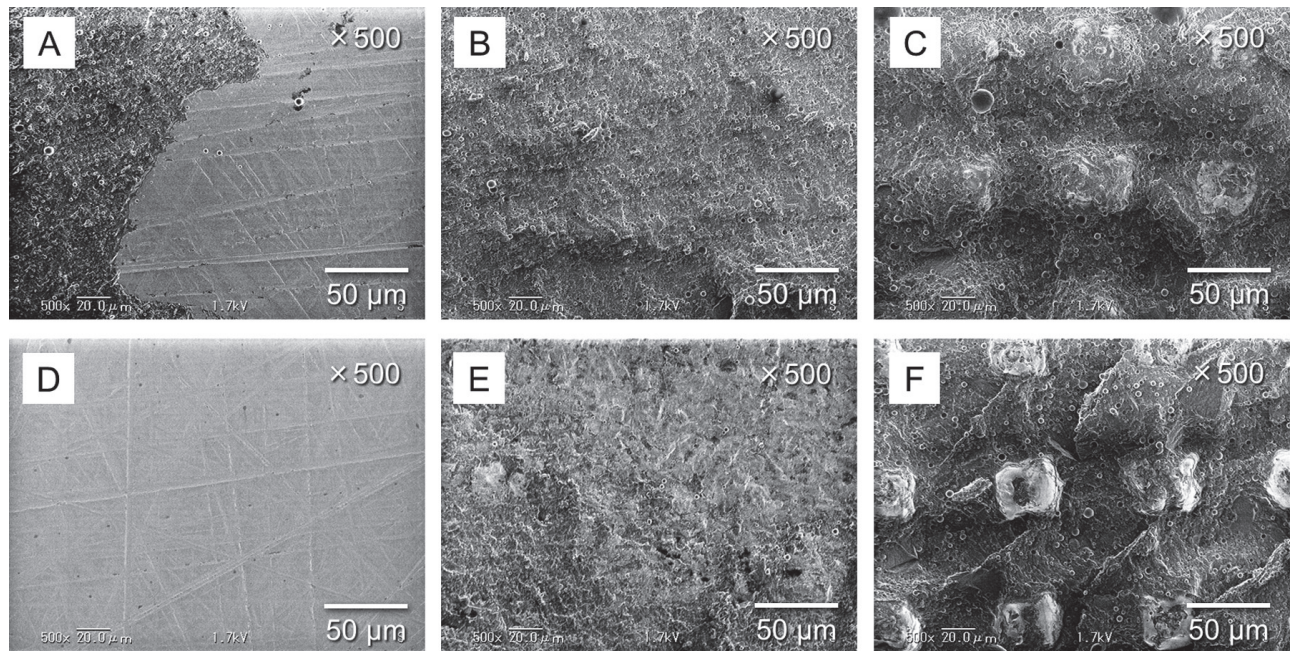

Fig. 5 SEM images of the debonded surface of Y-TZP veneered with indirect composite resin (500×); (A) non-treated, (B) alumina-blasted, and (C) with fabricated microslits before thermocycle, and (D) non-treated, (E) alumina-blasted, and (F) with fabricated microslits after thermocycle.
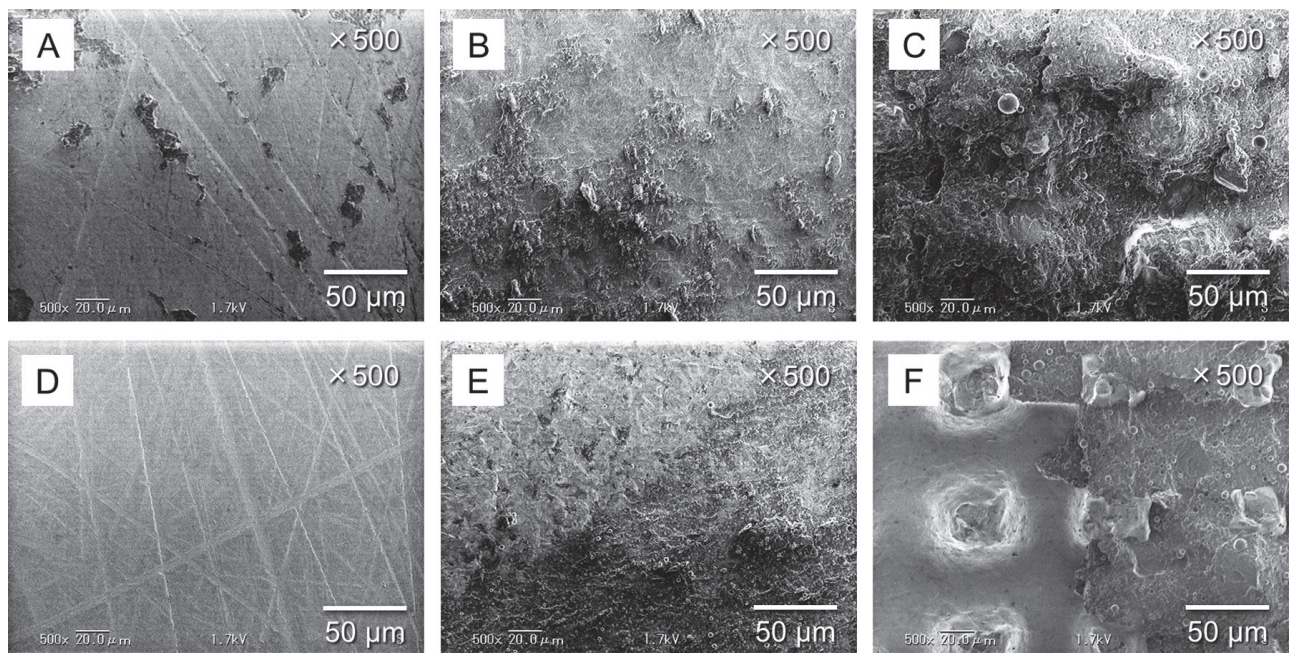

Fig. 6 SEM images of the debonded surface of Ce-TZP/A veneered with indirect composite resin (500×); (A) non-treated, (B) alumina-blasted, and (C) with fabricated microslits before thermocycle, and (D) non-treated, (E) alumina-blasted, and (F) with fabricated microslits after thermocycle. 
Table 7 Shear bond strengths of acrylic resin veneered to zirconia

\begin{tabular}{|c|c|c|c|c|c|c|c|c|}
\hline \multirow{2}{*}{ Groups } & \multicolumn{3}{|c|}{0 Thermocycles } & \multicolumn{3}{|c|}{ 20,000 Thermocycles } & \multirow{2}{*}{$\mathrm{S}$} & \multirow{2}{*}{ Reduction } \\
\hline & Mean & $\mathrm{SD}$ & Category & Mean & $\mathrm{SD}$ & Category & & \\
\hline \multicolumn{9}{|l|}{ Y-TZP } \\
\hline NT & 12.9 & 3.5 & a & 0 & 0 & & $\mathrm{~S}$ & $100 \%$ \\
\hline $\mathrm{AB}$ & 12.3 & 6.7 & a & 0 & 0 & & $\mathrm{~S}$ & $100 \%$ \\
\hline MS & 27.6 & 9.1 & $\mathrm{~b}$ & 17.5 & 7.1 & & & $36.6 \%$ \\
\hline \multicolumn{9}{|l|}{$\mathrm{Ce}-\mathrm{TZP} / \mathrm{A}$} \\
\hline $\mathrm{NT}$ & 16.1 & 2.7 & $\mathrm{~A}$ & 0 & 0 & & $\mathrm{~S}$ & $100 \%$ \\
\hline $\mathrm{AB}$ & 11.0 & 4.0 & B & 0 & 0 & & $\mathrm{~S}$ & $100 \%$ \\
\hline MS & 8.6 & 5.1 & $\mathrm{~B}$ & 0 & 0 & & $\mathrm{~S}$ & $100 \%$ \\
\hline
\end{tabular}

SD: Standard deviation; Category: Identical letters indicate that values are not statistically different ( $p>0.05)$; S: Significant difference between pre- and post-thermocycling bond strengths $(p<0.05)$.

Table 8 Failure modes of acrylic resin after shear bond testing

\begin{tabular}{|c|c|c|c|c|c|c|}
\hline \multirow{2}{*}{$\begin{array}{c}\text { Groups } \\
\text { Failure mode }\end{array}$} & \multicolumn{3}{|c|}{$\begin{array}{c}0 \\
\text { Thermocycles }\end{array}$} & \multicolumn{3}{|c|}{$\begin{array}{c}20,000 \\
\text { Thermocycles }\end{array}$} \\
\hline & $\mathrm{A}$ & $\mathrm{AC}$ & $\mathrm{C}$ & $\mathrm{A}$ & $\mathrm{AC}$ & C \\
\hline \multicolumn{7}{|l|}{ Y-TZP } \\
\hline NT & 10 & 0 & 0 & 10 & 0 & 0 \\
\hline $\mathrm{AB}$ & 10 & 0 & 0 & 10 & 0 & 0 \\
\hline MS & 2 & 8 & 0 & 2 & 8 & 0 \\
\hline \multicolumn{7}{|l|}{$\mathrm{Ce}-\mathrm{TZP} / \mathrm{A}$} \\
\hline NT & 10 & 0 & 0 & 10 & 0 & 0 \\
\hline $\mathrm{AB}$ & 10 & 0 & 0 & 10 & 0 & 0 \\
\hline MS & 10 & 0 & 0 & 10 & 0 & 0 \\
\hline
\end{tabular}

A: Adhesive failure at the zirconia-resin interface; C: Cohesive failure within the resin; AC: Combination of adhesive and cohesive failures.
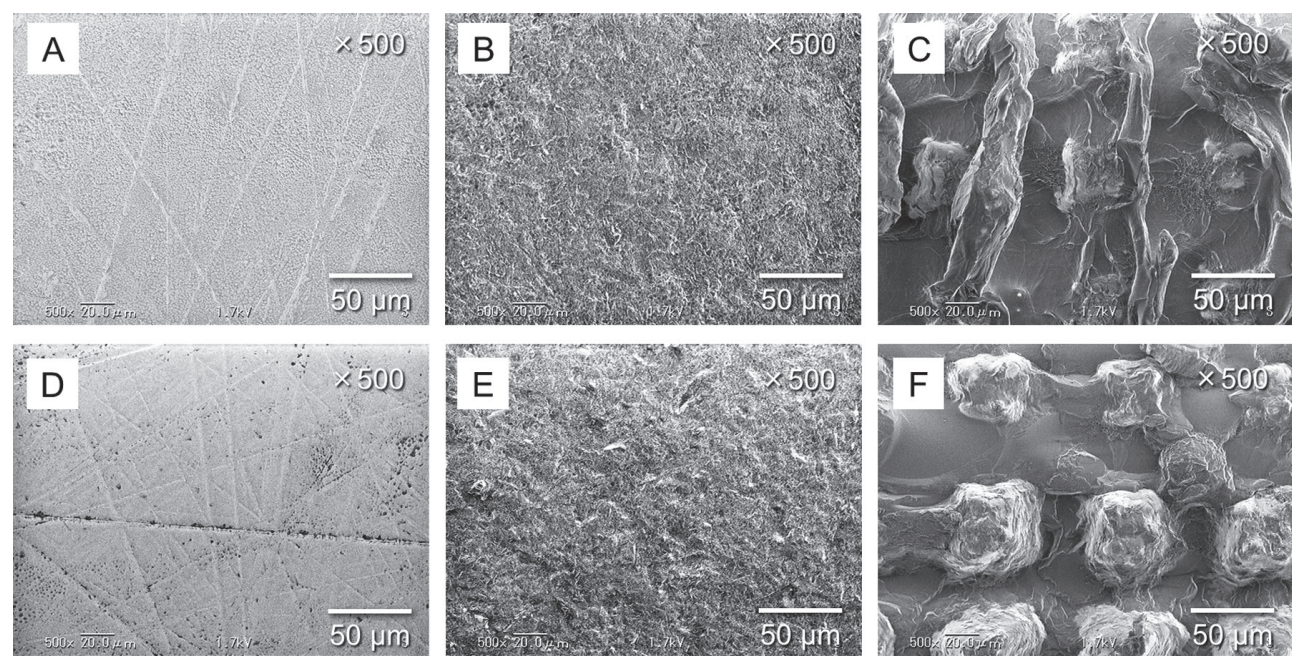

Fig. 7 SEM images of the debonded surface of Y-TZP veneered with acrylic resin (500×); (A) non-treated, (B) alumina-blasted, and (C) with fabricated microslits before thermocycle, and (D) non-treated, (E) alumina-blasted, and (F) with fabricated microslits after thermocycle. 

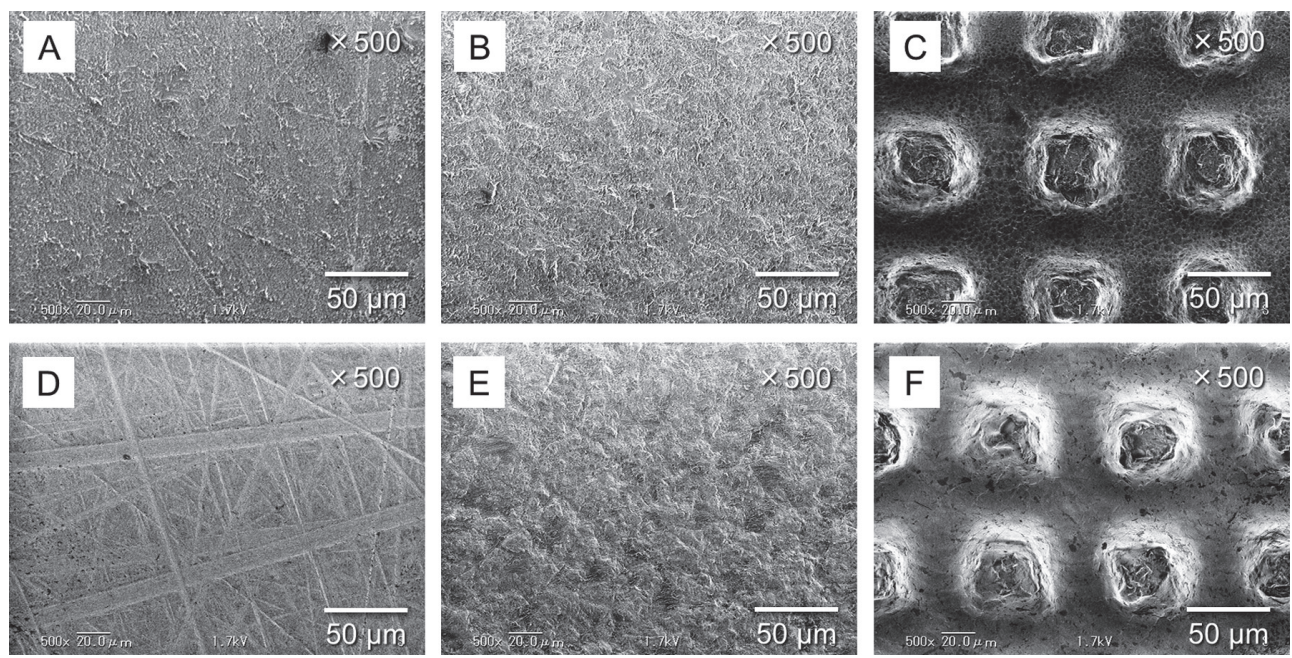

Fig. 8 SEM images of the debonded surface of Ce-TZP/A veneered with acrylic resin (500×); (A) non-treated, (B) alumina-blasted, and (C) with fabricated microslits before thermocycle, and (D) non-treated, (E) alumina-blasted, and (F) with fabricated microslits after thermocycle.

of just tens of micrometers. Even if microscale milling burs could be fabricated, severe wear of the bur would be expected, because the material considered in this study is fully sintered zirconia. Another approach is laser beam machining, which is commonly used in industry for the manufacture of injection molds, lettering and engravings, and can also produce microscopic surface texture on injection molds. This eliminates the need for burs. Therefore, this technology has the potential to make microslits on zirconia frames having complex shapes. For these reasons, laser beam machining was selected as the fabrication method for microretention features in this study. Microslits were fabricated on zirconia disks with flat surfaces and the SBSs of porcelain, indirect composite resin and acrylic resin veneered to the zirconia disks were measured. The microslits on the zirconia disks were fabricated in a simple reticular pattern because the purpose was to examine the SBS with or without microslits.

In general, since resins exhibit a relatively high level of water absorption, and their coefficient of thermal expansion (CTE) is higher than that of zirconia ${ }^{21)}$, a reduction in adhesion strength might be expected as a result of long-term exposure to moisture and significant temperature changes in the oral cavity. Therefore, the change in the SBS before and after thermal cycling was examined for indirect composite resin and acrylic resin. Because porcelain exhibits relatively low water absorption and its CTE is close to that of zirconia, no thermal cycling was performed on porcelain samples.

\section{Effects of microslits on porcelain veneered to zirconia}

We previously hypothesized that the strength of the bond between zirconia and porcelain would improve if the zirconia surface roughness was increased, and investigated the effect of alumina blasting with particles of different sizes on the SBS ${ }^{14)}$. However, there was no significant difference between the different particle sizes. Thus, the present study was carried out to investigate whether the bond strength could be improved by introducing a more geometric surface texture. After shear tests, there was no difference evident between the groups. However, in the MS group, SEM observations of both types of zirconia after the shear tests indicated that the microslits were still totally covered with the porcelain, i.e., the porcelain remaining on the zirconia may have been due to the microslits. Accordingly, this result indicated the microslits have the effect to hold the porcelain, although the SBS values were not increased by the microslits. Henriques et al. ${ }^{17)}$ reported that Nd:YAG laser surface structuring of zirconia increased the SBS with porcelain by up to $75 \%$. They produced holes in the zirconia surface with diameters of 25 and $50 \mu \mathrm{m}$, and depths of about 50 and $100 \mu \mathrm{m}$, respectively. Although the porcelain used in the study was veneered using an injection technique, which is different to our method, deeper microslits may be more effective for increasing the SBS.

The reason why there was no difference from NT despite the fact that all MS specimens underwent cohesive failure was that the mechanical strength of the porcelain penetrating the microslits was weaker than the adhesive strength. As a result, the strength of the porcelain was close to the SBS for the NT specimens. This may be due to the brittleness of the porcelain or the design of the microslits. Therefore, it may be necessary to strengthen the porcelain, or modify the microslits, for example by making them wider or changing their shape.

Effects of microslits on indirect composite resin bonding to zirconia

Previous studies have suggested that 10methacryloyloxydecyl dihydrogen phosphate (MDP) 


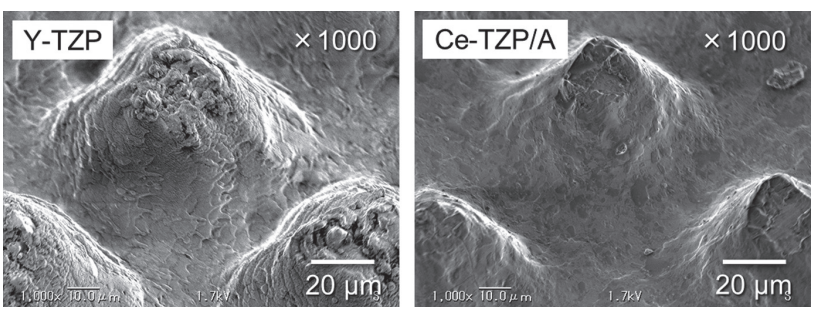

Fig. 9 SEM images of texture inside microslit (1,000×)

bonds chemically to zirconia ${ }^{9,22)}$. MDP consisting of phosphate monomers is effective to improve the bond strength between indirect composite resin and zirconia. Therefore, in this study, a primer including MDP was used as a chemical treatment to bond indirect composite resin to zirconia.

The SBSs of both types of zirconia of the NT group were significantly reduced after thermocycle, even though the SBSs before thermocycle were over 15.0 MPa. In contrast, both types of zirconia of the $\mathrm{AB}$ and $\mathrm{MS}$ groups exhibited measurable SBSs after thermocycle. This result demonstrates that the requirement for improvement of the bond durability is not only chemical treatment, but also surface roughening such as aluminablasting and the fabrication of microslits.

Furthermore, the SBS of Y-TZP of the MS group was significantly higher than that of the $A B$ group, regardless of thermocycling. However, Ce-TZP/A of the $\mathrm{AB}$ group before and after thermocycling showed the highest SBS. In addition, all specimens of the MS group with Y-TZP showed cohesive failure, whereas specimens on Ce-TZP/A showed adhesive and combined failure. Thus, the effectiveness of the microslits on Y-TZP could be greater than those on Ce-TZP/A. This is attributed to the difference in the structure between two types of zirconia. Figure 9 shows SEM images of texture inside microslit. According to the SEM images, the microslits on Y-TZP were relatively rougher than those on CeTZP/A. This difference might be due to difference of the crystal structure between two types of zirconia. Xie et $a l .{ }^{15)}$ reported that hot acid etching increased the surface roughness of Y-TZP, and improved the bonding with resin, which suggests that the surface roughness plays a significant role in the bonding. Therefore, the present result suggested that not only the design of the microstructure but also the texture inside the slit may affect the bond strength, and further investigation is needed.

Effects of microslits on acrylic resin bonding to zirconia In the present study, a primer containing MDP was applied to examine the effect of the microslits on the SBS between zirconia and acrylic resin. Several studies have reported that a primer that contain MDP is recommended for bonding zirconia with acrylic resin ${ }^{6,899}$. Accordingly, an alloy primer that contained MDP was used for this experiment, as with the third experiment.

The relationship between load and the flexibility of both Y-TZP and Co-Cr has been previously reported using a four-point bending test ${ }^{23)}$. The study reported that Y-TZP showed an equal maximum load but less flexibility than Co-Cr. However, the deformation of zirconia when it is packed in a flask and pressure is applied has not been clarified. Therefore, this study investigated the application of acrylic resin as a cast polymerization.

After thermocycles were applied to Y-TZP, the SBS of the specimens of the NT and AB groups could not be evaluated, because the resins were already debonded prior to the tests. In contrast, the MS group retained the resin on the Y-TZP surface after thermocycle, and the MS group exhibited a measurable SBS. In addition, under SEM observation, residual resin was found on some parts of the fracture surface of the MS group, before and after thermocycles. These results indicated that the microslits are superior for bond durability between Y-TZP and acrylic resin. Kawaguchi et al. ${ }^{24)}$ reported on the bonding of acrylic resin to $\mathrm{Ti}$ and $\mathrm{Co}-\mathrm{Cr}$ alloy with four surface treatments. The result of their studies indicated that the use of a primer containing MDP yielded the highest bond strength of acrylic resin to both $\mathrm{Ti}$ and $\mathrm{Co}-\mathrm{Cr}$ alloy after thermocycling. The bond strength of Ti is similar to that of the MS group of Y-TZP examined in the present study. Therefore, there is the possibility that the microslits on Y-TZP are effective for bonding acrylic resin to Y-TZP, as with the bond stability of removable partial dentures fabricated with acrylic resin and Ti.

However, among the Ce-TZP/A specimens, the SBS of the MS group before thermocycle was the lowest, followed by the $\mathrm{AB}$ group. Before shear tests, the specimens of the MS group were fractured by thermocycle stress, as with the other groups. In addition, SEM observations after shear tests showed that no residual resin was remained on the MS group zirconia surface. This is probably because, similar to the case for the indirect composite resin, the microslits on the CeTZP/A were smoother than those on the Y-TZP (Fig. 9). The surface texture inside the microslits may be an important factor in the case of an acrylic resin because the SBS after thermal cycling decreased significantly.

In this study, laser machining was performed in an area with a diameter of $5 \mathrm{~mm}$, and the processing time was about $5 \mathrm{~min}$. Since the machine used is for industrial applications, it is not yet easy to modify it for clinical use. It is necessary to improve the machining time and cost. However, we successfully demonstrated that a $\mathrm{Nd}: \mathrm{YVO}_{4}$ laser can engrave microscale structures in fully sintered zirconia. In particular, microslits on Y-TZP were shown to significantly improve the adhesion to indirect composite resin and acrylic resin. In the future, smaller machines are likely to be developed that are easier to use for dentistry, and can operate at lower cost.

Although the microslits had no effect on the bond strength with porcelain, they were particularly effective for Y-TZP for the two types of resin. This may be due to the difference in toughness between porcelain and 
resin. Because porcelain is brittle, the material that penetrates into the slits may be easy to break. Therefore, the slit shape may be inappropriate for porcelain. On the other hand, probably because the indirect composite resin and acrylic resin have a relatively high elongation at break, the microslits improved the bond strength. Although microslits were effective under some conditions, the SBS may be further improved by modifying their microstructure, for example by making them wider and deeper. In addition, from the results for indirect composite resin and acrylic resin veneered to $\mathrm{Ce}-\mathrm{TZP} / \mathrm{A}$, the texture inside the microslits may also be an important factor for improving the bond durability. Further investigation is needed to compare the influence of the microslit shape on the bond strength.

\section{CONCLUSIONS}

Within the limitations of the present study, the bond strength between porcelain and two types of zirconia was not improved by the use of microslits. In addition, the microslits on Ce-TZP/A did not improve the bond strength with acrylic resin, despite showing a better effect than non-treatment for adhesion to indirect composite resin. However, microslits on Y-TZP were found to improve the bond strength and adhesion durability in bonding with indirect composite resin or acrylic resin. The slit-like microstructure on zirconia partially improved the adhesive strength and durability. However, it is necessary to further investigate the effect of different microstructures on zirconia, for example wider and deeper slits or other shapes, on the bond strength between zirconia and porcelain or indirect composite resin or acrylic resin.

\section{ACKNOWLEDGMENTS}

This study was supported in part by a Grant-in-Aid for Scientific Research C (No. 16K11593) (2016-2018) from the Japan Society for the Promotion of Science (JSPS).

\section{REFERENCES}

1) Komine F, Blatz MB, Matsumura H. Current status of zirconia-based fixed restorations. J Oral Sci 2010; 52: 531-539.

2) Pang Z, Chughtai A, Sailer I, Zhang Y. A fractographic study of clinically retrieved zirconia-ceramic and metal-ceramic fixed dental prostheses. Dent Mater 2015; 31: 1198-1206.

3) Tamura Y, Kakuta K, Ogura H. Wear and mechanical properties of composite resins consisting of different filler particles. Odontology 2013; 101: 156-169.

4) Hamza TA, Attia MA, El-Hossary MM, Mosleh IE, Shokry TE, Wee AG. Flexural strength of small connector designs of zirconia-based partial fixed dental prostheses. J Prosthet Dent 2016; 115: 224-229.

5) Hagiwara $\mathrm{Y}$, Nakajima K. Application of $\mathrm{Ce}-\mathrm{TZP} / \mathrm{Al}_{2} \mathrm{O}_{3}$ nanocomposite to the framework of an implant-fixed complete dental prosthesis and a complete denture. J Prosthodont Res 2016; 60: 337-343.

6) Nakayama D, Koizumi H, Komine F, Blatz MB, Tanoue N, Matsumura H. Adhesive bonding of zirconia with single- liquid acidic primers and a tri-n-butylborane initiated acrylic resin. J Adhes Dent 2010; 12: 305-310.

7) Shimoe S, Tanoue N, Kusano K, Okazaki M, Satoda T. Influence of air-abrasion and subsequent heat treatment on bonding between zirconia framework material and indirect composites. Dent Mater J 2012; 31: 751-757.

8) Chiba Y, Shimoe S, Iwaguro S, Satoda T. Influence of surface treatments on bonding between zirconia ceramic material and denture base resin. JNADT. 2012; 33: 1-5.

9) Kubochi K, Komine F, Fushiki R, Yagawa S, Mori S, Matsumura $\mathrm{H}$. Shear bond strength of a denture base acrylic resin and gingiva-colored indirect composite material to zirconia ceramics. J Prosthodont Res 2017; 61: 149-157.

10) Lee CF, Pierpont HP, Strickler ER. The effect of bead attachment systems on casting patterns and resultant tensile bond strength of composite resin veneer cast restorations. J Prosthet Dent 1991; 66: 623-630.

11) Funaki K. Resin veneering procedure with the use of retention beads combined with dental adhesive. J Jpn Prosthodont Soc 1994; 38: 211-220.

12) Guess PC, Kuliš A, Witkowski S, Wolkewitz M, Zhang Y, Strub JR. Shear bond strengths between different zirconia cores and veneering ceramics and their susceptibility to thermocycling. Dent Mater 2008; 24: 1556-1567.

13) Nakamura T, Wakabayashi K, Zaima C, Nishida H, Kinuta $\mathrm{S}$, Yatani H. Tensile bond strength between tooth-colored porcelain and sandblasted zirconia framework. J Prosthodont Res 2009; 53: 116-119.

14) Iwaguro $S$, Shimoe $S$, Chiba $Y$, Ohkura $H$, Satoda $T$. Influence of alumina-blasting and heating rate on the bond strength between zirconia and veneering porcelain. JNADT 2013; 34: 8-14.

15) Xie H, Chen C, Dai W, Chen G, Zhang F. In vitro short-term bonding performance of zirconia treated with hot acid etching and primer conditioning etching and primer conditioning. Dent Mater J 2013; 32: 928-938.

16) Nakamura T, Sugano T, Usami H, Wakabayashi K, Ohnishi $\mathrm{H}$, Sekino T, et al. Shear bond strength of veneering porcelain to porous zirconia. Dent Mater J 2014; 33: 220-225.

17) Henriques B, Fabris D, Souza JCM, Silva FS, Carvalho O, Fredel MC, et al. Bond strength enhancement of zirconiaporcelain interfaces via Nd:YAG laser surface structuring. J Mech Behav Biomed Mater 2018; 81: 161-167.

18) Noda M, Okuda Y, Tsuruki J, Minesaki Y, Takenouchi Y, Ban S. Surface damages of zirconia by Nd:YAG dental laser irradiation. Dent Mater J 2010; 29: 536-541.

19) Kazama-Koide M, Ohkuma K, Ogura H, Miyagawa Y. A new method for fabricating zirconia copings using a Nd:YVO nanosecond laser. Dent Mater J 2014; 33: 422-429.

20) Kato K, Sakai H, Nomura H, Kondo Y, Yatani H, Yamashita A. Microbeading resin-bonded retainers Part 1. tensile bond strengths and durability. J Jpn Prosthodont Soc 1989; 33: 1398-1407.

21) Rajan G, Shouha P, Ellakwa A, Bhowmik K, Xi J, Prusty G. Evaluation of the physical properties of dental resin composites using optical fiber sensing technology. Dent Mater 2016; 32: 1113-1123.

22) Kern M, Wegner SM. Bonding to zirconia ceramic: adhesion methods and their durability. Dent Mater 1998; 14: 64-71.

23) Sanaoka S, Iwahori M, Goto T, Watanabe K, Higashino $\mathrm{Y}$, Imadu $\mathrm{H}$, et al. Application of dental zirconia to major connector. J Gifu Dent Soc 2010; 37: 41-46.

24) Kawaguchi T, Shimizu H, Lassila LVJ, Vallittu PK, Takahashi Y. Effect of surface preparation on the bond strength of heat-polymerized denture base resin to commercially pure titanium and cobalt-chromium alloy. Dent Mater J 2011; 30: 143-150. 